\title{
Monitoring Neutrophil Elastase and Cathepsin G Activity in Human Sputum Samples
}

\author{
Dario L. Frey ${ }^{*}, 1,2$, Matteo Guerra ${ }^{*}, 1,2,3,4$, Marcus A. Mall ${ }^{1,2,5,6,7}$, Carsten Schultz ${ }^{1,3,8}$ \\ ${ }^{1}$ Translational Lung Research Center Heidelberg (TLRC), German Center for Lung Research (DZL) ${ }^{2}$ Dept. of Translational Pulmonology, University \\ of Heidelberg ${ }^{3}$ Molecular Medicine Partnership Unit (MMPU), European Molecular Biology Laboratory (EMBL), University of Heidelberg ${ }^{4}$ Faculty of \\ Biosciences, Collaboration for Joint Ph.D. Degree between EMBL and Heidelberg University, University of Heidelberg ${ }^{5}$ Dept. of Pediatric Pulmonology, \\ Immunology and Critical Care Medicine, Charité - Universitätsmedizin Berlin ${ }^{6}$ Berlin Institute of Health (BIH) ${ }^{7}$ German Center for Lung Research (DZL), \\ Associated Partner Site, Berlin ${ }^{8}$ Dept. of Chemical Physiology and Biochemistry, Oregon Health and Science University \\ *These authors contributed equally
}

\section{Corresponding Authors}

Marcus A. Mall

marcus.mall@charite.de

Carsten Schultz

schulcar@ohsu.edu

\section{Citation}

Frey, D.L., Guerra, M., Mall, M.A.,

Schultz, C. Monitoring Neutrophil

Elastase and Cathepsin G Activity in Human Sputum Samples. J. Vis. Exp.

(171), e62193, doi:10.3791/62193

(2021).

\section{Date Published}

May 21, 2021

DOI

$10.3791 / 62193$

URL

jove.com/video/62193

\section{Abstract}

Proteases are regulators of countless physiological processes and the precise investigation of their activities remains an intriguing biomedical challenge. Among the $\sim 600$ proteases encoded by the human genome, neutrophil serine proteases (NSPs) are thoroughly investigated for their involvement in the onset and progression of inflammatory conditions including respiratory diseases. Uniquely, secreted NSPs not only diffuse within extracellular fluids but also localize to plasma membranes. During neutrophil extracellular trap (NETs) formation, NSPs become an integral part of the secreted chromatin. Such complex behavior renders the understanding of NSPs pathophysiology a challenging task. Here, detailed protocols are shown to visualize, quantify and discriminate free and membrane-bound neutrophil elastase (NE) and cathepsin G (CG) activities in sputum samples. NE and CG are NSPs whose activities have pleiotropic roles in the pathogenesis of cystic fibrosis (CF) and chronic obstructive pulmonary disease (COPD): they promote tissue remodeling, regulate downstream immune responses and correlate with lung disease severity. The protocols show how to separate fluid and cellular fraction, as well as the isolation of neutrophils from human sputum for enzymatic activity quantification via small-molecule Förster resonance energy transfer-based (FRET) reporters. To gather specific insights into the relative role of NE and CG activities, a FRET readout can be measured by different technologies: i) in vitro plate reader measurements allow for high-throughput and bulk detection of protease activity; ii) confocal microscopy spatiotemporally resolves membrane-bound activity at the cell surface; iii) smallmolecule FRET flow cytometry enables for the rapid evaluation of anti-inflammatory treatments via single-cell protease activity quantification and phenotyping. The 
implementation of such methods opens the doors to explore NSPs pathobiology and their potential as biomarkers of disease severity for CF and COPD. Given their standardization potential, their robust readout and simplicity of transfer, the described techniques are immediately shareable for implementation across research and diagnostic laboratories.

\section{Introduction}

Neutrophil elastase (NE), cathepsin G (CG), proteinase 3 (PR3) and neutrophil serine protease 4 (NSP4) are the four neutrophil serine proteases (NSPs) ${ }^{1}$. They are stored, together with myeloperoxidase, within neutrophil primary or azurophilic granules. Due to their elevated proteolytic content, the secretion of primary granules is tightly regulated and neutrophils have to be sequentially challenged with priming and activating stimuli ${ }^{2}$.

Inside the phagolysosome, NSPs function as intracellular bactericidal agents ${ }^{3}$. When secreted, NSPs become strong mediators of inflammation: they cleave cytokines and surface receptors, activating parallel pro-inflammatory pathways ${ }^{3}$. Importantly, inflammatory conditions feature an uncontrolled NSPs secretion. For example, within inflamed airways, excessive NE activity causes mucus hypersecretion, goblet cells metaplasia, CFTR inactivation and extracellular matrix remodeling ${ }^{4,5}$. Cathepsin $\mathrm{G}$ participates in inflammation as well: it specifically cleaves and activates two components of the IL-1 family, IL-36 $\alpha$ and IL- $36 \beta^{6}$. In concert with $\mathrm{NE}, \mathrm{CG}$ cleaves protease-activated receptors on the airway epithelium and also activates TNF- $\alpha$ and IL-1 $\beta$.

Endogenous anti-proteases such as alpha-1-antitrypsin, alpha-1-antichymotrypsin and the secretory leukocyte protease inhibitor regulate neutrophil elastase and cathepsin $G$ activity ${ }^{5}$. However, over the course of lung disease progression, the continuous secretion of proteases exceeds stoichiometrically the anti-protease shield, leading to nonresolving neutrophilia in the airways, inflammation worsening and tissue damage ${ }^{5,7}$. Although NE concentration and activity in soluble fractions of patient airways has been shown to be a promising biomarker of disease severity ${ }^{8}$, NE and CG also associate to the neutrophil plasma membrane and to extracellular DNA via electrostatic interactions ${ }^{9,10}$ where they become less accessible to anti-proteases. Importantly, preclinical studies defined a scenario where cell surface-associated protease activity appears earlier and/or independently of its soluble counterpart ${ }^{4,11}$. In fact, to become detectable, free protease activity first needs to overwhelm the anti-protease shield. Instead, at the cell surface, membrane-bound protease activity remains at least partially intact due to the inaccessibility of large inhibitors to the cell plasma membrane ${ }^{12}$. Such complex protease behavior has important consequences on neutrophil-mediated inflammation onset and propagation, and therefore needs to be investigated with precise and informative tools.

Over the years, Förster resonance energy transfer (FRET)based probes found numerous biomedical applications as tools that efficiently and rapidly assess a specific protease activity in human samples ${ }^{13}$. To function, protease reporters are composed of a recognition motif (i.e., a peptide), which is recognized by the target enzyme and rely on FRET, a 
physical process where, upon excitation, a donor fluorophore transfers energy to an acceptor molecule. The processing operated by the enzyme on the reporter, namely the cleavage of the recognition part, results in the acceptor to diffuse away from the donor: the enzyme activity is therefore measured as a time-dependent change in the donor over the acceptor fluorescence. Such read-out is self-normalizing and ratiometric, hence only marginally affected by environmental conditions such as $\mathrm{pH}$ and local probe concentration. NEmo- $1^{14}$ and SSAM $^{15}$ are FRET probes that report specifically on NE and CG activity, respectively. However, such reporters do not localize specifically to any cellular compartment, therefore they are employed to monitor the protease activity present in human fluids. In order to monitor protease activity in a spatially localized fashion, we and others developed FRET probes that associate to subcellular components via molecular tags $14,15,16,17,18,19$. Such a synthetic strategy allowed the development of NEmo-2 and mSAM, two FRET probes equipped with lipid anchors that localize to the plasma membrane. These reporters fueled a deeper understanding of NE and CG proteases in cystic fibrosis and chronic obstructive pulmonary diseases ${ }^{14,15}$.

Here, detailed protocols are provided for the visualization and quantification of soluble and membrane-bound NE and CG activities in human sputum by means of NEmo and SAM series of FRET probes. To address diverse aspects of NSPs pathophysiology and provide an array of methods that can be employed according to the user-specific need, the analysis via fluorescence spectroscopy, fluorescence microscopy and flow cytometry are shown.

\section{Protocol}

The following protocols describe analysis performed on human sputum. Human sample handling was approved by the ethics committee of the University of Heidelberg and written informed consent was obtained from all patients or their parents/legal guardians (S-370/2011) and healthy controls (S-046/2009).

NOTE: The following protocols describe the sample preparation and the quantification of neutrophil serine proteases (NSPs) activity. The experimental procedures presented herein focus on human sputum and neutrophil elastase $14,20,21$ (NE) or cathepsin $G^{15}$ (CG) activity measurement. However, slight adaptations in the sample preparation protocol render the analysis of blood-derived cells and tumor homogenates possible. In addition, matrix metalloproteinase 12 and cathepsin $S$ activities can be investigated similarly by means of dedicated FRET probes $^{22,23,24,25,26}$.

\section{Sample preparation: cell isolation and supernatant separation}

NOTE: If possible, the treatment of sputum should be carried out within 120 min after expectoration and the sputum should be stored on ice until further processing.

1. If spontaneous expectoration of sputum is not possible, induce sputum as described previously ${ }^{19}$. Briefly, inhale $200 \mu \mathrm{g}$ of the $\beta$-2-receptor-antagonist salbutamol before starting the sputum induction procedure. Afterwards, inhale a hypertonic (6\%) saline solution for 15 min using a nebulizer. Collect the expectorated sputum in a Petri dish.

2. Separate the mucus clumps from the saliva into a Petri dish with the help of a pipette tip.

3. Weigh the mucus. 
NOTE: The average weight of a mucus samples is $0.8 \mathrm{~g}$ (varying between $0.1 \mathrm{~g}$ and $5 \mathrm{~g}$ ); $0.1 \mathrm{~g}$ are usually sufficient to perform the mentioned procedures.

4. Add 4 parts (v/w) of $10 \%$ Sputolysin (in PBS) to sputum (for example: $4 \mathrm{~mL}$ of $10 \%$ Sputolysin for each gram of sputum).

CAUTION: Sputolysin is composed of concentrated dithiothreitol in phosphate buffer, therefore handle it with care.

5. Incubate the mixture at room temperature (RT) on a rocking shaker for $15 \mathrm{~min}$ to dissolve the mucus. For safety reasons, place the shaker into a fume hood.

6. Quench the reaction by adding the same volume of cold PBS (for example: $1 \mathrm{~mL}$ of cold PBS for each $\mathrm{mL}$ of $10 \%$ Sputolysin).

7. Mix by pipetting to obtain a homogenous solution.

8. Filter the mixture through a $100 \mu \mathrm{m}$ nylon cell strainer into a $50 \mathrm{~mL}$ tube.

9. Repeat the filtration step through a $40 \mu \mathrm{m}$ nylon cell strainer.

10. Centrifuge the solution for $10 \mathrm{~min}$ at $300 \mathrm{xg}$ at $4{ }^{\circ} \mathrm{C}$.

11. Transfer the supernatant fraction carefully into a fresh tube and store it on ice.

NOTE: The supernatant fractions can be stored at $-20^{\circ} \mathrm{C}$ or $-80^{\circ} \mathrm{C}$ until further analysis.

12. Gently resuspend the cell pellet in $500 \mu \mathrm{L}$ of cold PBS and place it on ice.

NOTE: The cell fraction must be processed immediately.

\section{Neutrophil serine protease activity measurement}

NOTE: Here, different methods are introduced to quantify NSPs activity by means of FRET reporters. The choice of the technology is dictated by the specific biomedical question and purpose of the experiment. The probes presented were extensively tested for their specificity against a set of lung relevant enzymes ${ }^{14,15}$. Although the probes are specific toward their target enzyme, always check the probe specificity on the clinical sample of interest. This can be achieved by incubating the sample with a specific protease inhibitor prior to probe addition, which should abolish any increase in the D/A ratio.

1. Soluble NSPs activity quantification via fluorimeter or plate reader assay

NOTE: Protease activity in soluble fractions of the sample can be detected with any instrument capable of fluorescence detection.

1. Thaw enzymes on ice.

2. Until use, keep NE and CG in acidic storage buffer (50 mM sodium acetate, $200 \mathrm{mM} \mathrm{NaCl}, \mathrm{pH}$ 5.5) to prevent self-cleavage.

3. To set up an enzyme standard curve, prepare a 1:2 serial dilution of enzyme (33.9 - $0.271 \mathrm{nM}$ for $\mathrm{NE} ; 42.6-0.333 \mathrm{nM}$ for $\mathrm{CG}$ ) in activation buffer (10 mM Tris- $\mathrm{HCl}, 500 \mathrm{mM} \mathrm{NaCl}$ at $\mathrm{pH}$ 7.5). The activation buffer has a neutral $\mathrm{pH}$ and therefore enables enzymatic catalysis to occur efficiently.

1. To prepare the highest standard concentration (NE concentration: $33.9 \mathrm{nM}$ ), dilute $1 \mu \mathrm{L}$ of $\mathrm{NE}$ $(33.9 \mu \mathrm{M})$ in $999 \mu \mathrm{L}$ of activation buffer.

2. To prepare the second standard (NE concentration: $16.95 \mathrm{nM}$ ), mix $200 \mu \mathrm{L}$ of the first dilution with $200 \mu \mathrm{L}$ of activation buffer.

3. Proceed accordingly to prepare the remaining $1: 2$ dilutions. 
4. The last standard, which is the blank, is composed of pure activation buffer. The measurement of technical duplicates or triplicates is recommended. During the standard and sample preparation, try to keep vials on ice.

4. In parallel to the standard preparation, dilute sputum samples in activation buffer. Dilute the human samples before assessing their protease activity to remain in the linear range of increase of reporter signal (donor/acceptor ratio). If patient samples were left undiluted, the cleavage would happen too rapidly for a reliable fitting. Since healthy donor sputum contains fewer active proteases compared to samples from CF and COPD patients, different dilutions are generally performed (1:10 for healthy sputum supernatant, 1:20-500 for sputum supernatant of COPD or CF patients).

NOTE: In order to quantitatively measure protease activity in samples where their concentration is unknown, a standard curve with known enzyme concentrations needs to be measured in parallel, ideally on the same plate. The concentration of active enzyme in human sputum is calculated via interpolating the slopes measured in human sputum samples with the ones measured with the standard curves.

5. Before preparing the samples for measurement, set up the instrument. Set the excitation wavelength for NE FRET probe (NEmo-1 ${ }^{14}$ ) to $354 \mathrm{~nm}$, and set the detection wavelength to $400 \mathrm{~nm}$ for the donor and $490 \mathrm{~nm}$ for the acceptor. Set the excitation wavelength for CG FRET probe $\left(\mathrm{SSAM}^{15}\right)$ to 405 $\mathrm{nm}$, and set the emission to 485 (donor) and $580 \mathrm{~nm}$ (acceptor).

6. Add $40 \mu \mathrm{L}$ of samples, standard or blank into the wells of a black 96 -well half area plate.

7. To prepare the master mix containing the reporters (concentration of the reporter in the master mix: $10 \mu \mathrm{M}$ ), dilute the probe stock (1 $\mathrm{mM}$ in DMSO) 1:100 in activation buffer. Prepare the needed master mix volume by multiplying $10 \mu \mathrm{L} x$ the number of required plate wells. To reach the optimal final concentration $(2 \mu \mathrm{M})$ for fluorescence measurement of NEmo-1 and SSAM reporters, add $10 \mu \mathrm{L}$ of the master mix to each well (containing 40 $\mu \mathrm{L}$ of either sample, standard or blank) and start the readout. NEmo-1 and SSAM reporters will therefore monitor soluble neutrophil elastase and cathepsin G activity, respectively.

NOTE: If a reagent injector is not available, make sure to start the readout as soon as possible after reporter addition to the samples.

8. Start the plate reader measurement and record the donor/acceptor ratio increase every $60-90$ seconds for at least $20 \mathrm{~min}$ or until the increase in the signal reaches a plateau.

9. Once data are exported, calculate the donor/ acceptor ratio (D/A ratio) by dividing the donor relative fluorescence units (RFU) with the acceptor RFU for each time point and sample.

10. Calculate the $D / A$ ratio mean and standard deviation of each sample.

11. Determine the slope within the linear growth of the D/A ratio change. The slope is an indicator of the enzyme cleavage rate for a FRET probe. Calculate 
the concentration of active enzyme in sputum by fitting the linear regression slopes derived from the human samples with the ones calculated from the enzyme standard.

2. Membrane-bound NSPs activity quantification via fluorimeter or plate reader assay

1. Isolate sputum cells as described above. Resuspend $3 \times 10^{4}$ cells in a volume of $40 \mu \mathrm{L}$ of PBS. Add the cells to plate reader well.

2. Set up the instrument. Set the excitation wavelength for membrane-bound NE FRET probe (NEmo-2 ${ }^{14}$ ) to $405 \mathrm{~nm}$, and set the detection wavelength to $485 \mathrm{~nm}$ for the donor and $580 \mathrm{~nm}$ for the acceptor. Set the excitation wavelength for membrane-bound CG FRET probe $\left(\mathrm{mSAM}^{15}\right)$ to $405 \mathrm{~nm}$, and set the emission to 485 (donor) and $580 \mathrm{~nm}$ (acceptor).

3. To prepare the master mix containing the reporters, dilute the probe stock in activation buffer to a concentration of $10 \mu \mathrm{M}$. Prepare the needed master mix volume by multiplying $10 \mu \mathrm{L} X$ the number of required plate wells. To reach the optimal final concentration $(2 \mu \mathrm{M})$ for fluorescence measurement of NEmo-2 and mSAM reporters, add $10 \mu \mathrm{L}$ of the master mix to each well (containing $40 \mu \mathrm{L}$ of either sample, standard or blank) and start the readout. NEmo-2 and mSAM reporters will therefore monitor membrane-bound neutrophil elastase and cathepsin $\mathrm{G}$ activity, respectively.

NOTE: A cellular negative control can be used, for example, cells which do not actively secrete NSPs. For example, the incubation of the reporters with $3 \times 10^{4}$ leukocyte progenitors HL-60 promyelocytic cells represents a valid cleavage negative control.
4. Record change in donor/acceptor ratio for at least 20 min or until the increase of the signal reaches a plateau. Analyze the data as described above.

3. Membrane-bound NSPs activity measurement via fluorescence microscopy

1. Determine the number of conditions which need to be analyzed.

NOTE: For each sputum sample measurement, the preparation and analysis of additional positive (PC) and negative (NC) control is recommended.

2. For each measurement, resuspend $3 \times 10^{4}$ sputum cells in a volume of $50 \mu \mathrm{L}$ PBS in a $1.5 \mathrm{~mL}$ tube.

3. As a negative control, incubate sputum cells with a specific inhibitor (Sivelestat, specific NE inhibitor, or cathepsin G inhibitor I, specific CG inhibitor, at a final concentration of $100 \mu \mathrm{M})$. Incubate for $10 \mathrm{~min}$ at RT.

4. As positive control, incubate sputum cells with the appropriate enzyme (NE or CG at $340 \mathrm{nM}$ or $200 \mathrm{nM}$, respectively) for $10 \mathrm{~min}$ at $\mathrm{RT}$.

5. Add $50 \mu \mathrm{L}$ of PBS containing the FRET reporter and a nuclear stain (in a 1:1000 final dilution) to each tube (positive control-treated cells, negative controltreated cells and untreated cells) in order to reach a probe final concentration of $2 \mu \mathrm{M}$. Incubate for $10-20$ $\min$ at RT.

IMPORTANT: The addition of a nuclear stain facilitates fluorescence microscopy imaging as it allows to search for sputum cells of interest without using the FRET probe channels and therefore to avoid reporter bleaching. In addition, the DNA stain permits to segment sputum cells according to the shape of their nucleus. For example, neutrophils can be easily identified by their multilobular nuclei. Also, 
additional information about the viability of the cells can be retrieved (neutrophils with a more segmented nucleus are more likely to be alive).

NOTE: In addition to the membrane-bound one, the activity of DNA-bound NE or CG in human sputum can be measured in the same way by means of $\mathrm{H}$ NE and H-CG, extracellular DNA-associating FRET probes $^{27}$. When preparing the master mix, H-NE and $\mathrm{H}-\mathrm{CG}$ can be added at the concentration of $10 \mu \mathrm{M}$ and incubated with sputum prior to cytospin and slide preparation. The D/A ratio quantification on extracellular DNA proceeds identically to NEmo-2 and mSAM, with the only difference that extracellular DNA aggregates are segmented instead of single cells ${ }^{27}$.

6. Quench the reaction by adding $100 \mu \mathrm{L}$ of ice-cold PBS, and transfer samples on ice.

7. Cytospin the mixture on microscopy slides, air dry, fix with ice cold methanol $10 \%$ for $10 \mathrm{~min}$, air dry and mount with an appropriate mounting medium.

NOTE: The microscopy slides can be stored at $4{ }^{\circ} \mathrm{C}$ in the dark for up to one month until further analysis.

8. Acquire microscopy images using a confocal microscope with a PL APO 40x or 63x oil objective. To increase image quality and reduce acquisition time a sequential image acquisition mode is recommended.

1. Image the nuclear stain first via $633 \mathrm{~nm}$ excitation with the helium-neon-laser line and record its emission between 650 and $715 \mathrm{~nm}$.
2. Record the donor (coumarin 343) of the FRET reporter between 470 and $510 \mathrm{~nm}$ upon excitation at $458 \mathrm{~nm}$ with an argon laser. Acquire the sensitized acceptor (5,6-TAMRA) emission between 570 and $610 \mathrm{~nm}$ after sole donor excitation.

3. Record the direct acceptor emission in a separate channel between 470 and $510 \mathrm{~nm}$ upon acceptor optimal excitation at $561 \mathrm{~nm}$ using the diode pumped solid state (DPSS) laser.

4. Set the pinhole at the beginning of the experiment and maintain during the course of the imaging session.

NOTE: Due to their small size, the use of a $40 x$ or $63 x$ microscope objective is recommended to visualize neutrophils properly. Usually, images are acquired in several consecutive channels, starting with the longer excitation wavelength to prevent photobleaching of the reporter during acquisition.

9. Image at least 100 cells per condition for conclusive statistics. For microscopy images analysis use an appropriate software: segment the cells and calculate the D/A ratio on a pixel-by-pixel basis, afterwards calculate the mean or median of a given region of interest which is selected manually by the user. For representative results see Figure 1. 

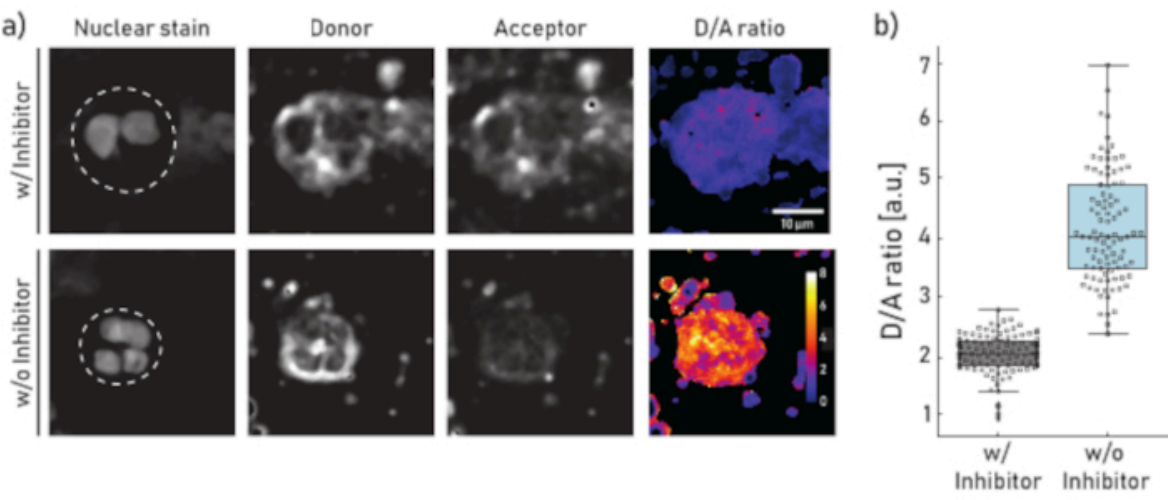

Figure 1: Representative images and quantification of membrane-bound NE activity on neutrophils isolated from CF patient sputum. a) Representative confocal microscopy images of neutrophils pre-incubated (top panel) for 10 min with $100 \mu \mathrm{M}$ of Sivelestat $(\mathrm{w} /)$ or left untreated (w/o) (bottom panel) before reporter NEmo-2 $(2 \mu \mathrm{M})$ addition. The first column from the left shows the nuclear stain, the second the donor channel, the third the acceptor channel and the last the calculated D/A ratio obtained by dividing donor and acceptor channels on a pixel-by-pixel basis. The borders of the region of interest (single neutrophil) are depicted as dashed line. Scale $(10 \mu \mathrm{m})$ and calibration bars (D/A ratio) are indicated. b) Box-and dot-plots showing the D/A ratio of sputum neutrophils from a representative CF patient. Cells incubated with inhibitor and untreated cells are shown in grey and blue, respectively. Each dot represents one cell $(\mathrm{N}: \mathrm{w} /$ inhibitor $=113$ and $\mathrm{w} / \mathrm{o}$ inhibitor =96). Please click here to view a larger version of this figure.

4. Membrane-bound NSPs activity measurement via flow cytometry

1. Resuspend $1 \times 10^{6}$ cells in $100 \mu \mathrm{L}$ of PBS in a $5 \mathrm{~mL}$ FACS polystyrene round-bottom tube and place the tube on ice.

2. To gate sputum neutrophils, use the following antibodies: CD14 (1:50), CD16 (1:50), CD45 (1:33) and CD66b (1:50). Prepare sufficient master mix for all samples. Place master mix on ice in the dark.

3. Set up the gating strategy as described in Figure 2. The neutrophils are gated as 7AAD ${ }^{-} \mathrm{CD}_{45^{+}} \mathrm{CD}^{-} 4^{-} \mathrm{CD} 16^{+} \mathrm{CD}^{-} 6 \mathrm{~b}^{+}$events. The gated events will be then analyzed for their membrane-bound protease activity for their donor $\left(\lambda_{\text {exc }}=405 \mathrm{~nm}, \lambda_{\mathrm{em}}=450 / 50 \mathrm{~nm}\right)$ and acceptor $\left(\lambda_{\text {exc }}=405 \mathrm{~nm}, \lambda_{\text {em }}=585 / 42 \mathrm{~nm}\right)$ mean fluorescent intensities (MFIs).

4. Add $2 \mu \mathrm{L}$ of FcBlock to each sample, and incubate for 5 min at RT.

5. Add the chosen antibodies to each tube, and incubate for 30 min on ice in the dark.

6. Wash cells by adding $2 \mathrm{~mL}$ of cold PBS, and centrifuge for $5 \mathrm{~min}$ at $300 \mathrm{x} \mathrm{g}$ and $4{ }^{\circ} \mathrm{C}$. Discard supernatant and resuspend cells in $200 \mu \mathrm{L}$ of cold PBS. 
7. Split the $200 \mu \mathrm{L}$ in two tubes with $100 \mu \mathrm{L}$ each and add $5 \mu \mathrm{L}$ of cell viability staining solution in each tube. Place tubes on ice.

8. Add an appropriate specific NSP inhibitor (for NE use Sivelestat at $225 \mu \mathrm{M}$ final concentration, for CG use cathepsin $\mathrm{G}$ Inhibitor I at $100 \mu \mathrm{M}$ ) to the negative control (NC) test tube. Incubate the samples at RT for $10 \mathrm{~min}$ in the dark.

9. Add $100 \mu \mathrm{L}$ of cold PBS to the sample, filter it through a $40 \mu \mathrm{m}$ filter in a clean FACS tube to prevent clogging of the instrument.

10. Add the reporter (for NE, NEmo-2 at a final concentration of $4 \mu \mathrm{M}$, for $\mathrm{CG}, \mathrm{mSAM}$ at a final concentration of $2 \mu \mathrm{M}$ ) to the NC sample, gently vortex the tube.

11. Start acquiring cells incubated with the specific inhibitor to slightly adjust, if necessary, the gates as well as the reporter PMTs' voltages.

12. Record at least 1000 neutrophils. Keep the sample at room temperature.

NOTE: Although more events can be recorded, 1000 cells ensure a good compromise between proper statistics and recording time.
13. Proceed with the following tubes (untreated sputum samples) accordingly.

14. To record changes in the D/A ratio due to membrane-bound protease activity, record 1000 neutrophils from each tube every 5 to $10 \mathrm{~min}$.

NOTE: After successful FRET reporter cleavage, the donor channel MFIs intensity will increase over time. The acceptor channel MFIs intensity should decrease or remain constant over time.

15. Calculate the FRET ratio by dividing the donor by the acceptor channel values for the samples measured on the gated viable single neutrophils.

16. Normalize sample-measurements by dividing them with the corresponding $0 \mathrm{~min}$ time point (for representative results and analysis see Figure 2). NOTE: The recording of at least two time points (i.e., 0 and $10 \mathrm{~min}$ ) is necessary for a dynamic measurement of D/A ratio change. To normalize the activity measurement for each sample, the $D /$ A ratio measured in later time points (e.g., $10 \mathrm{~min}$ ) is divided by the ratio calculated immediately after probe addition (0 $\mathrm{min})$. 

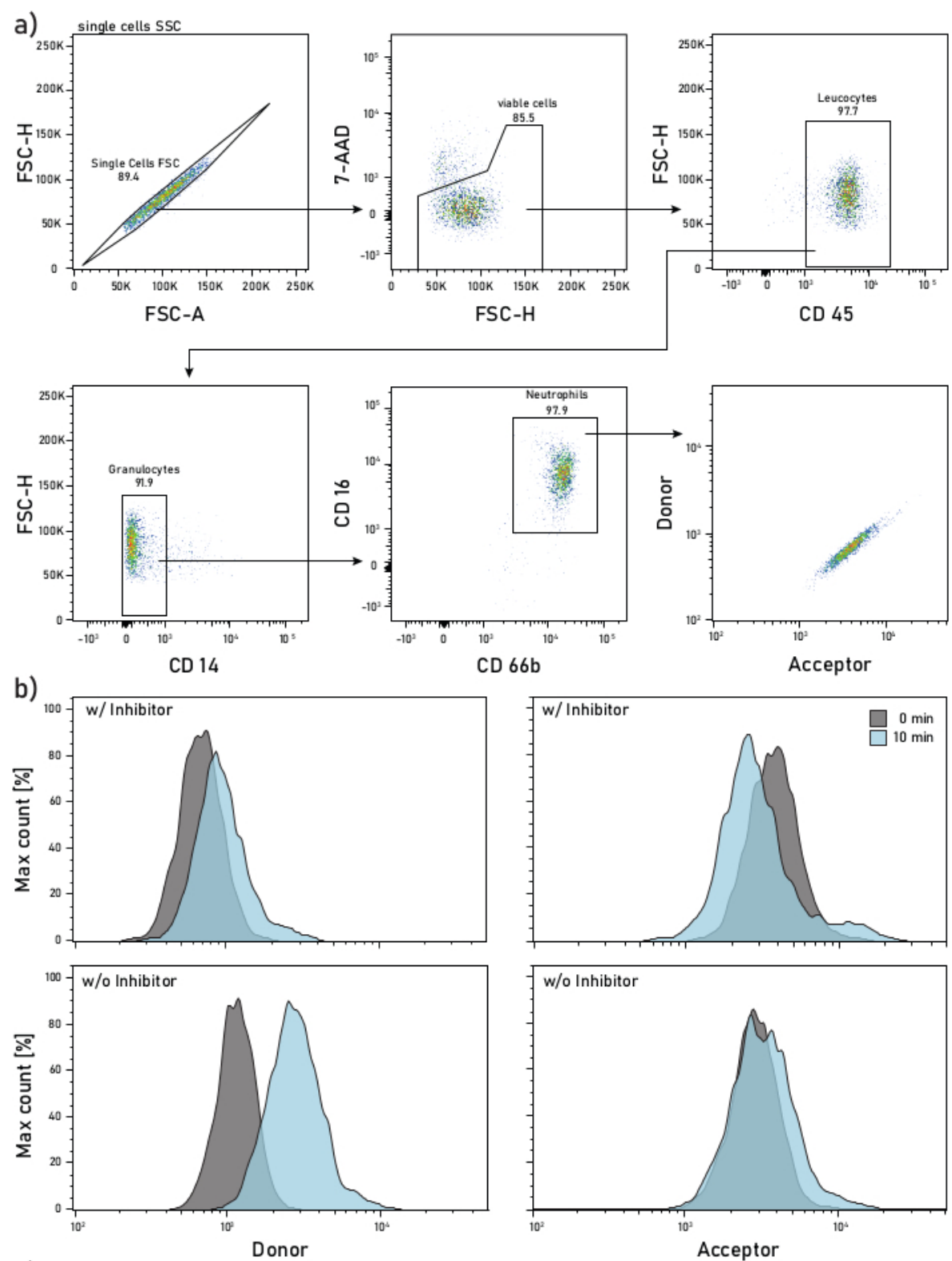

c)

\begin{tabular}{|c|c|c|c|c|c|}
\hline & $\begin{array}{c}\text { time } \\
\text { min }\end{array}$ & $\begin{array}{l}\text { Donor } \\
\text { MFI }\end{array}$ & $\begin{array}{c}\text { Acceptor } \\
\text { MFI }\end{array}$ & $\begin{array}{l}\mathrm{D} / \mathrm{A} \\
\text { ratio }\end{array}$ & $\begin{array}{c}\text { D/A ratio } \\
\text { norm. }\end{array}$ \\
\hline \multirow{6}{*}{$\begin{array}{l}\frac{2}{0} \\
\frac{0}{0} \\
\frac{1}{5} \\
\frac{5}{3}\end{array}$} & 0 & $503 * 188$ & $6639 \pm 2641$ & 0.08 & 1.00 \\
\hline & 3 & $479 \pm 195$ & $5752 \pm 2449$ & 0.08 & 1.00 \\
\hline & 5 & $505 \pm 214$ & $5783 \pm 2731$ & 0.09 & 1.13 \\
\hline & 10 & $516 \pm 206$ & $5363 \pm 2322$ & 0.10 & 1.25 \\
\hline & 15 & $571 \pm 260$ & $5622 \pm 3269$ & 0.10 & 1.25 \\
\hline & 20 & $584 \pm 255$ & $5476 \pm 2870$ & 0.11 & 1.37 \\
\hline \multirow{7}{*}{ 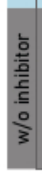 } & 0 & $544 \pm 177$ & $6654 \div 2225$ & 0.08 & 1.00 \\
\hline & 3 & $2393 \pm 910$ & $6854 \pm 3118$ & 0.35 & 4.37 \\
\hline & 5 & $2923 \pm 1134$ & $6480 \pm 2909$ & 0.45 & 5.62 \\
\hline & 10 & $3594 \pm 1390$ & $7079 \pm 3210$ & 0.51 & 6.37 \\
\hline & 15 & $3801 \pm 1510$ & $7075 \pm 3144$ & 0.54 & 6.75 \\
\hline & 20 & $3909 \pm 1552$ & $7081 \pm 3343$ & 0.55 & 6.87 \\
\hline & & \multicolumn{2}{|c|}{ raw values } & \multicolumn{2}{|c|}{ calculated } \\
\hline
\end{tabular}

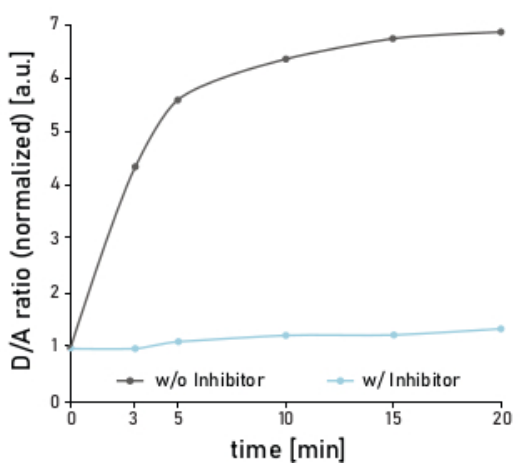

Figure 2: Gating strategy and representative plots of membrane-bound NE activity measured on neutrophils isolated from CF patient sputum. a) To gate sputum neutrophils the following antibodies are used: CD14 (1:50), CD16 
(1:50), CD45 (1:33) and CD66b (1:50). The neutrophils are gated as 7-AAD-CD45 ${ }^{+} \mathrm{CD}^{-} 4^{-} \mathrm{CD} 16^{+} \mathrm{CD}^{-} 6 \mathrm{~b}^{+}$events. The gated events are analyzed for their donor $\left(\lambda_{\text {exc }}=405 \mathrm{~nm}, \lambda_{\mathrm{em}}=450 / 50 \mathrm{~nm}\right)$ and acceptor $\left(\lambda_{\mathrm{exc}}=405 \mathrm{~nm}, \lambda_{\mathrm{em}}=585 / 42\right.$ $\mathrm{nm}$ ) mean fluorescence intensities (MFIs). b) Representative histograms of CF sputum neutrophils analyzed for their membrane-bound NE activity. The left column shows the donor signal, the right column shows the acceptor signal. The top row shows mean fluorescence intensities of cells treated with Sivelestat (w/) for 10 min before addition of the reporter. The bottom row shows untreated (w/o) cells whose reporter fluorescence is measured immediately ( 0 min, grey) and 10 min (blue) after reporter addition. Neutrophils are gated according to the strategy shown in panel a. c) The data table shows a representative dataset consisting of raw MFIs for the donor and acceptor signal on neutrophils measured over several time points (0-3-5-10-15-20 $\mathrm{min}$ ) as well as the calculated D/A ratio. The D/A ratio can be normalized, i.e., to the 0 min time point (white font). 0 min indicates a recording done as soon as possible after reporter addition to the flow tube with stained sputum cells. MFIs data are shown as mean \pm standard deviation for 1000 neutrophils. Please click here to view a larger version of this figure.

\section{Representative Results}

The results shown in Figure 1a illustrate a representative microscopy dataset. The nuclear signal is used to identify neutrophils by their characteristic segmented nuclei. The region of interest $(\mathrm{ROI})$ is selected manually (dashed line in Figure 1a). The D/A ratio image is calculated by dividing the intensities of the donor channel by the intensities of the acceptor channel on a pixel-by-pixel basis. In the last step the average D/A ratio per cell $(\mathrm{ROI})$ is calculated. In Figure 1b each dot represents the mean of one ROI (neutrophil). It is recommended to image and evaluate about 100 cells per condition.

A representative flow cytometry gating strategy is shown in Figure 2a. Such gating allows to discriminate and study sputum neutrophils. To avoid fluorescence spillover or compensation artifacts, it is recommended to dedicate a laser line (e.g., blue laser) to the FRET probe fluorescence detection. Flow cytometry fluorescence compensation should be performed for antibodies and not for the FRET probe. Figure $\mathbf{2 b}$ depicts the MFI distribution at 0 and 10 min after reporter addition. Each row in Figure $2 \mathrm{c}$ indicates the mean donor and acceptor MFIs values for 1000 sputum neutrophils. The D/A ratio is calculated by dividing the donor and acceptor MFIs. The time course in Figure $\mathbf{2 c}$ on the right side shows the progression of the measurement: after a rapid initial increase, the D/A ratio reaches a plateau, according to the activity of the membrane-bound enzyme.

\section{Discussion}

The reported protocols explain different approaches to quantify the activity of neutrophil elastase and cathepsin G in human sputum samples. Critical points for a successful enzyme activity measurement are the i) precise timing and standardization of the operative procedure and ii) the use of reliable negative and positive controls. If these conditions are met, the described methods are not limited to sputum but can also be easily adapted to the analysis of protease activity in blood, bronchoalveolar lavage fluids and tissue sections or homogenates.

Each of the three techniques has its strengths and limitations, which often complement each other. For example, flow cytometry allows for the rapid analysis of rare cell 
populations as well as cell phenotyping but lacks spatial resolution information, which can be achieved by microscopy. Instead, plate reader measurements permit for the parallel assessment of several samples or conditions in a highthroughput fashion. Since fresh sputum cells cannot be frozen and stored, the three methods require that samples must be processed rapidly after expectoration. This limits the flexibility or the throughput of the membrane-bound activity measurements. The development of a flow cytometry protocol that allows to fix cells after probe addition and enzymatic cleavage would open to the parallel measurement of a higher number of tubes. Moreover, particular attention should be paid to the handling and storage of the FRET probes. In fact, some aminoacids present in the peptide substrate, such as methionine, undergo oxidation which leads to decreased reporter sensitivity. To increase the reporter's shelf life (estimated of about three months at $20^{\circ} \mathrm{C}$ ), they can be stored in small volume aliquots $(1-2 \mu \mathrm{L})$ under inert gas such as Nitrogen or Argon.

In CF and other chronic inflammatory lung diseases it is important to detect the inflammation as early as possible, and reliable biomarkers have the potential to achieve such a goal. The possibility to detect surface-bound NSPs activity, which has been shown to be harmful for the surrounding tissue, also in conditions when there is no or little free NE activity, adds another level of valuable information, which can be hardly achieved by means of other existing methods ${ }^{4,11}$.

The reporters can be used to study the link of membranebound associated NSP activity with severity and progression of lung disease, especially at its early-onset. The methods can be utilized to monitor treatment efficacy (e.g., antiinflammatory treatments or highly effective CFTR modulators and potentiators ${ }^{28}$ ) and investigate the resulting dampening of neutrophil-driven inflammation. In addition, the protocols are based on non-invasive sample procedures which carry very low risk for the patient and, therefore, can be used on a very broad scale and open the doors to numerous exciting applications.

\section{Disclosures}

The authors declare no conflict of interests.

\section{Acknowledgments}

This project was supported by grants from the German Ministry for Education and Research (FKZ 82DZL004A1 to M.A.M) and the German Research Foundation (SFB-TR84TP B08 to M.A.M). Work described in this manuscript was supported by the German Center of Lung Research (DZL) and the EMBL Heidelberg through a PhD fellowship for M.G. We thank J. Schatterny, S. Butz and H. Scheuermann for expert technical assistance.

\section{References}

1. Korkmaz, B., Moreau, T., Gauthier, F. Neutrophil elastase, proteinase 3 and cathepsin G: Physicochemical properties, activity and physiopathological functions. Biochimie. 90 (2), 227-242 (2008).

2. Sheshachalam, A., Srivastava, N., Mitchell, T., Lacy, P., Eitzen, G. Granule Protein Processing and Regulated Secretion in Neutrophils. Frontiers in Immunology. 5, 448 (2014).

3. Pham, C.T.N. Neutrophil serine proteases: Specific regulators of inflammation. Nature Reviews Immunology. 6 (7), 541-550 (2006). 
4. Gehrig, S. et al. Lack of neutrophil elastase reduces inflammation, mucus hypersecretion, and emphysema, but not mucus obstruction, in mice with cystic fibrosislike lung disease. American Journal of Respiratory and Critical Care Medicine. 189 (9), 1082-1092 (2014).

5. McKelvey, M.C., Weldon, S., McAuley, D.F., Mall, M.A., Taggart, C.C. Targeting proteases in cystic fibrosis lung disease paradigms, progress, and potential. American Journal of Respiratory and Critical Care Medicine. 201 (2), 141-147 (2020).

6. Clancy, D.M. et al. Extracellular Neutrophil Proteases Are Efficient Regulators of IL-1, IL-33, and IL-36 Cytokine Activity but Poor Effectors of Microbial Killing. Cell Reports. 22 (11), 2937-2950 (2018).

7. Giacalone, V.D., Margaroli, C., Mall, M.A., Tirouvanziam, R. Neutrophil adaptations upon recruitment to the lung: New concepts and implications for homeostasis and disease. International Journal of Molecular Sciences. 21 (3), 1-21 (2020).

8. Sly, P.D. et al. Risk Factors for Bronchiectasis in Children with Cystic Fibrosis. New England Journal of Medicine. 368 (21), 1963-1970 (2013).

9. Owen, C.A., Campbell, M.A., Sannes, P.L., Boukedes, S.S., Campbell, E.J. Cell surface-bound elastase and cathepsin G on human neutrophils: A novel, nonoxidative mechanism by which neutrophils focus and preserve catalytic activity of serine proteinases. Journal of Cell Biology. 131 (3), 775-789 (1995).

10. Brinkmann, V. et al. Neutrophil Extracellular Traps Kill Bacteria. Science. 303 (5663), 1532-1535 (2004).

11. Margaroli, C. et al. Elastase Exocytosis by Airway Neutrophils Associates with Early Lung Damage in Cystic
Fibrosis Children. American Journal of Respiratory and Critical Care Medicine. (2018).

12. Owen, C A et al. "Cell surface-bound elastase and cathepsin G on human neutrophils: a novel, nonoxidative mechanism by which neutrophils focus and preserve catalytic activity of serine proteinases." The Journal of cell biology. vol. 131,3 (1995): 775-89. (1995).

13. Garland, M., Yim, J.J., Bogyo, M. A Bright Future for Precision Medicine: Advances in Fluorescent Chemical Probe Design and Their Clinical Application. Cell chemical biology. 23 (1), 122-136 (2016).

14. Gehrig, S., Mall, M.A., Schultz, C. Spatially resolved monitoring of neutrophil elastase activity with ratiometric fluorescent reporters. Angewandte Chemie International Edition. 51 (25), 6258-6261 (2012).

15. Guerra, M. et al. Cathepsin G Activity as a New Marker for Detecting Airway Inflammation by Microscopy and Flow Cytometry. ACS Central Science. 5 (3), 539-548 (2019).

16. Hu, H.Y. et al. In vivo imaging of mouse tumors by a lipidated cathepsin S substrate. Angewandte Chemie International Edition. 53 (29), 7669-7673 (2014).

17. Korkmaz, B. et al. Measuring elastase, proteinase 3 and cathepsin $G$ activities at the surface of human neutrophils with fluorescence resonance energy transfer substrates. Nature Protocols. 3 (6), 991-1000 (2008).

18. Craven, T.H. et al. Super-silent FRET Sensor Enables Live Cell Imaging and Flow Cytometric Stratification of Intracellular Serine Protease Activity in Neutrophils. Scientific Reports. 8 (1), 13490 (2018).

19. $\mathrm{Mu}$, J. et al. A small-molecule fret reporter for the real-time visualization of cell-surface proteolytic enzyme 
functions. Angewandte Chemie - International Edition. 53 (52), 14357-14362 (2014).

20. Hagner, M. et al. New method for rapid and dynamic quantification of elastase activity on sputum neutrophils from patients with cystic fibrosis using flow cytometry. European Respiratory Journal. 55 (4), 1902355 (2020).

21. Dittrich, A.S. et al. Elastase activity on sputum neutrophils correlates with severity of lung disease in cystic fibrosis. European Respiratory Journal. 1701910 (2018).

22. Cobos-Correa, A., Trojanek, J.B., Diemer, S., Mall, M.A., Schultz, C. Membrane-bound FRET probe visualizes MMP12 activity in pulmonary inflammation. Nature Chemical Biology. 5 (9), 628-630 (2009).

23. Hu, H.-Y. et al. FRET-based and other fluorescent proteinase probes. Biotechnology Journal. 9 (2), 266-281 (2014).

24. Trojanek, J.B. et al. Airway mucus obstruction triggers macrophage activation and matrix metalloproteinase 12-dependent emphysema. American Journal of Respiratory Cell and Molecular Biology. 51 (5), 709-720 (2014).

25. Wagner, C.J., Schultz, C., Mall, M.A. Neutrophil elastase and matrix metalloproteinase 12 in cystic fibrosis lung disease. Molecular and Cellular Pediatrics. 3 (1), 25 (2016).

26. Gaggar, A. et al. The role of matrix metalloproteinases in cystic fibrosis lung disease. The European respiratory journal. 38 (3), 721-7 (2011).

27. Guerra, M. et al. Protease FRET Reporters Targeting Neutrophil Extracellular Traps. Journal of the American Chemical Society. 142 (48), 20299-20305 (2020).
28. Middleton, P.G. et al. Elexacaftor-Tezacaftor-Ivacaftor for Cystic Fibrosis with a Single Phe508del Allele. New England Journal of Medicine. 381 (19), 1809-1819 (2019). 